\title{
Gastronomía clínica. Excelente alternativa para mejorar la nutrición hospitalaria
}

\author{
Clinical Gastronomy. Excellent alternative to improve hospital nutrition \\ Gastronomia clínica. Excelente alternativa para melhorar a nutrição hospitalar
}

Juan Carlos de la Cruz Castillo Pineda ${ }^{*}$

\author{
Recibido: 4 de julio de 2020. Aceptado para publicación: 3 de agosto de 2020 \\ Publicado en línea: 16 de septiembre de 2020 \\ Doi: https://doi.org/10.35454/rncm.v3n2.178
}

\section{Resumen}

La malnutrición en los hospitales es un problema endémico que incrementa la morbilidad, mortalidad y estancia hospitalaria. Existen muchos factores que la favorecen como son la inanición del enfermo (incluso antes de su ingreso), los procedimientos a los que es sometido, y la mala calidad y presentación de los alimentos que reciben los pacientes; propiciada, además por errores en la prescripción, transcripción, elaboración y distribución por parte del médico, nutricionista y preparador de alimentos, entre otros.

En consecuencia, se observa baja ingesta y desperdicio de alimentos (mayor de $40 \%$ ) lo que no solo genera deterioro del estado general del enfermo sino alta insatisfacción con el servicio de alimentos. Por lo anterior, es necesario posicionar la gastronomía clínica dentro de los equipos de salud que junto con el nutricionista deberá diseñar y desarrollar nuevos menús y estrategias como por ejemplo el menú a la carta, el room service, la calidad y presentación de los platillos, el registro del consumo de los alimentos, aplicación de encuestas de satisfacción a los usuarios, la observación de las características de los desperdicios para, con toda esta información valiosa desarrollar un programa de mejora continua en beneficio de los enfermos.

Palabras clave: gastronomía, malnutrición, servicio de alimentación en hospital.

\section{Summary}

Malnutrition in hospitals is an endemic problem that increases morbidity, mortality and hospital stay. There are many factors involved in its development, such as patient starvation, even before admission, the type of procedure performed, and the poor quality and presentation of the meals received, which in turn might be caused by errors in prescription, transcription, preparation, and distribution by the physician, nutritionist, or cook.

Consequently, intake decreases, and more than $40 \%$ of the food is wasted, which only leads to patient deterioration and increased dissatisfaction with the hospital catering service. Therefore, it is essential to position clinical gastronomy within the health teams so that together with the nutritionist, they develop new menus and strategies, such as à la carte menu or room service, ensure the quality and presentation of the dishes, record food consumption, user satisfaction, and waste characteristics to develop a continuous improvement program for the benefit of patients.

Keywords: Gastronomy; Malnutrition; Food service hospital.

\section{Resumo}

A desnutrição nos hospitais é um problema endêmico que aumenta a morbilidade, mortalidade e a permanência hospitalar. Existem muitos fatores que a favorecem, como a inanição do paciente (mesmo antes da admissão), os procedimentos a que ele é submetido e a má qualidade e apresentação dos alimentos que os pacientes recebem; originada, também, por erros na prescrição, transcrição, preparação e distribuição por médico, nutricionista e preparador de alimentos, entre outros.

Consequentemente, observa-se baixa ingestão e desperdício de alimentos (superior a 40\%), o que não só origina deterioração no estado geral do paciente, mas também alta insatisfação com o serviço de alimentação. Portanto, é necessário posicionar a gastronomia clínica nas equipes de saúde que, juntamente com o nutricionista, devem elaborar e desenvolver novos menus e estratégias como menu a la carte, o serviço de quarto, qualidade e apresentação dos pratos, o registo do consumo de alimentos, a aplicação de questionários de satisfação aos usuário, a observação das características dos esperdícios para, com todas essas informações valiosas desenvolver um programa de melhoria contínua em benefício dos pacientes.

Palavras-chave: gastronomia, desnutrição, serviço de alimentação no hospital. 
"You are not to permit your patient to encounter the terrible consequences of starvation because he does not ask for nutriment. Gentlemen, these results are due to good feeding. When I am gone, you may be at a loss for an epitaph for me. I give it to you in these words. He fed fevers" (1).

Robert Graves Dublin, 1843

\section{INTRODUCCIÓN}

Todo ser vivo tiene la insustituible necesidad de alimentarse del medio que lo rodea para la obtención de energía y sustratos para un adecuado metabolismo. Esto lo hacen todas las especies vivas, pero solo el hombre aprendió a $\operatorname{cocinar}_{\text {los alimentos }}{ }^{(2)}$. En un principio los ingerían crudos, secos o en estado de putrefacción, siendo la mayoría de origen vegetal. Cuando agrega los alimentos de origen animal concurre un desarrollo cerebral importante, controla el fuego, nace la agricultura y evoluciona la preparación de alimentos progresivamente.

El hombre empieza a desarrollar preferencias por las fuentes de comida, según agrada al paladar, luego los combina, adereza, guisa y así, aparece el arte culinario y la gastronomía. Por tanto, el comer no solo satisface las necesidades energéticas, sino que se convierte en un acto placentero, socializante que debe tenerse en cuenta en la consejería nutricional y en la alimentación de enfermos.

El término Gastronomía se deriva del griego

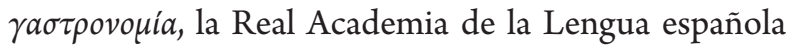
lo define como el arte de preparar una buena comida ${ }^{(3)}$. El grado gastronómico de un país constituye un índice importante de la evolución de un grupo humano y más si se enfoca en la atención de la población más vulnerable como son los enfermos. El verdadero reto para el nutriólogo es poder ofrecer una alimentación no solo suficiente, completa, balanceada, adecuada, inocua y accesible, sino que su presentación llene los sentidos: vista, olfato, gusto, temperatura y textura, para estimular de esta manera su consumo.

La preocupación por una dieta saludable y adecuada a la salud y enfermedad no es nueva. En el siglo V a.C. en el Corpus Hipocraticum existen varios tratados acerca de la dieta (la dieta entendida no solo como el acto de comer, sino también de mantener una actividad física cotidiana y ordenada, la higiene y el vestido) para sanos y enfermos, destacando la importancia de que el médico conociera a fondo los alimentos y bebidas, y sus efectos sobre el paciente ${ }^{(4)}$.
Butterworth (1974) en su artículo clásico "The skeleton in the hospital closet" destaca la poca atención que se tiene a la alimentación en los enfermos y afirma que la malnutrición en hospitales es inducida principalmente por el médico y enlista 14 prácticas atentatorias contra el estado nutricional; entre las cuales destaca la falta de registro de ingresos alimentarios, y la pobre comunicación entre el médico y el nutricionista que afectan la calidad de atención en el servicio de alimentos, entre otros $^{(5)}$. Simon Alisson (1996) por su parte, afirma que el modelo de atención de los servicios de alimentos se ha desvinculado de la atención a los enfermos, se ha generalizado de tal manera, que se brinda igual en residencias de ancianos, ejército o internados lo que ha deteriorado la calidad de atención y ha tenido repercusión en la morbilidad, mortalidad, estancia hospitalaria y costos de atención ${ }^{(1)}$. El problema está en que la alimentación de enfermos no es un servicio de hotelería asistencial, sino un proceso individualizado en donde la preparación, transporte y presentación de los alimentos debe garantizar que el paciente lo consuma en su totalidad, le sea agradable y que sea supervisado por el nutricionista $^{(1)}$. El paciente requiere una alimentación adecuada, perfeccionada con la técnica gastronómica en óptimas condiciones de contenido y estética.

Por otra parte, es frecuente que no haya suficiente personal de nutricionistas para supervisar la ingesta alimentaria, y para diseñar planes de mejora en el suministro de alimentos para los enfermos, lo que ocasiona inseguridad alimentaria en hospitales públicos y privados ${ }^{(6)}$ con efectos negativos en los egresos hospitalarios, principalmente en los grupos vulnerables como ancianos, pacientes con cáncer y con polimorbilidades agravando así la malnutrición existente al ingreso hospitalario ${ }^{(7)}$. Diversos estudios han estimado la malnutrición hospitalaria al ingreso entre $15 \%$ y $60 \%{ }^{(8)}$. El estudio ENHOLA (2016) demostró una frecuencia de malnutrición en hospitales latinoamericanos de $45 \%$, documentándose que a 40,1 \% de los participantes en el estudio no le gustaba la comida hospitalaria y de estos, se calculó, un riesgo de malnutrición de 3,6 (IC: 3,42 - 3,9 $)^{(9)}$. En otros estudios se demostró que los pacientes consumían menos de $70 \%$ de su requerimiento diario, probablemente, por ser una dieta impuesta sin tener en cuenta las preferencias, hábitos, horarios y costumbres del enfermo ${ }^{(10)}$.

Simzari et al. demostraron, en 120 pacientes, insatisfacción con los alimentos en $75 \%$ de la población estudiada, una ingesta aproximada de $2 / 3$ del requerimiento calculado y un desperdicio superior a $30 \%{ }^{(7)}$. 
Otros estudios reportan $38 \%$ de desperdicio a pesar de que el cálculo de energía y proteínas fue el adecuado y la prescripción correcta ${ }^{(11)}$, por lo que hay que considerar otros factores relacionados con el proceso y distribución de los alimentos, además de los factores propios de la enfermedad.

Se entiende por desperdicio del plato la cantidad de alimento no ingerido por el paciente ${ }^{(12)}$ cuyas causas pueden ser: prescripción inadecuada respecto al estado del paciente, anorexia, dieta no acorde con los hábitos y costumbres, horarios, temperatura, sabor, ninguna supervisión del plato que se entrega al paciente, etc., por lo que el monitoreo del desperdicio de alimentos ya sea por inspección visual o peso en gramos, así como la aplicación de cuestionarios a los pacientes ayuda a visibilizar el problema y mejorar la atención alimentaria ${ }^{(12)}$. Además, grupos de pacientes con enfermedades cardiovasculares, respiratorias y cáncer, que debido a su situación no comen, se desnutren con mayor facilidad ${ }^{(13)}$.

\section{SERVICIOS DE ALIMENTOS EN EL HOSPITAL}

En la Edad Media, los hospitales y servicios de alimentación de enfermos fueron organizados inicialmente por órdenes religiosas, con la finalidad de dar sustento y abrigo en especial a los pobres, por lo que la calidad y cantidad de comida estaba limitada por las circunstancias económicas, religiosas y patrones culturales de lo que era bueno o malo para el enfermo ${ }^{(14)}$. Es a finales del siglo XVIII cuando empieza una mejor organización principalmente por razones de economía más que de salud y hasta mediados del siglo XIX, cuando Florence Nightingale señala la presencia de la desnutrición hospitalaria y hace énfasis en que la alimentación formaba parte del cuidado de enfermería; sin embargo, en la actualidad no ocurre así en muchos de los hospitales, relegando la preparación y distribución de alimentos a personal sin la preparación adecuada lo que demerita la calidad del proceso $^{(1,15)}$.

En 1899 se funda la American Hospital Association que transforma el hospital de asistencia de pobres, en el moderno sistema de hospitales en donde el cuidado de la alimentación hospitalaria se considera importante. En los años 30, del siglo pasado, en Canadá se forman los primeros servicios de dietética y aparece el interés por el buen servicio de catering, la satisfacción del cliente y el control de presupuesto con sus grandes ventajas y nuevos retos ${ }^{(16)}$.

Las guías de ESPEN, 2017, definen el término hospital catering o suministro hospitalario de alimentos como el conjunto de procesos que garantizan el servir una variedad de alimentos adecuados y adaptados a los enfermos, con diversas cualidades nutricionales y con dietas especiales en cuanto a consistencia, textura, y variaciones acordes con las patologías. Los alimentos deberían estar disponibles las 24 horas del día, su presentación apetitosa, suficiente y con alternativas de alta densidad energética y proteica ${ }^{(17)}$. Es la gastronomía que se incorpora al cuidado de la salud, interdisciplinar, centrada en la alimentación, que considera los hábitos y costumbres del paciente, lugar de origen y aplica las técnicas y procedimientos de cocción de los alimentos para hacerlos comestibles, digeribles, inocuos y evidentemente agradables a la vista del comensal y en este caso, del enfermo, como cita Grande Covian "las personas comerán lo que deben, si les gusta” ${ }^{(18,19)}$.

El término de Gastronomía Clínica se ha usado desde hace décadas. En España Geles Duch y en Brasil Daniel Magnoni han trabajado arduamente en el desarrollo y difusión de esta a través de publicaciones y cursos, en donde se destaca que "no por la condición de enfermedad se le debe negar el placer de una buena comida al paciente" ${ }^{20-22)}$ y más si la alimentación es un derecho humano ${ }^{(23,24)}$ y reflejo de la autonomía de toda persona humana. El trabajo es interdisciplinario, el médico precisa un diagnóstico clínico con su propuesta terapéutica y en conjunto con la nutricionista determinan el riesgo nutricional del paciente a través de una adecuada evaluación nutricional, pero no solo de los aspectos antropométricos, bioquímicos, clínicos y dietéticos, sino que además debe verificar si existe alguna limitación del olfato, gusto, apetito, saciedad, etc., los hábitos y costumbres de alimentación, así como preferencias gastronómicas. Se establece un plan nutricional que se analiza con profesionales de la gastronomía y se encuentra la mejor alternativa que garantice una adecuada composición nutricional y realce del sabor y presentación de los alimentos ${ }^{(20)}$. El proceso deberá incluir también, la evaluación de lo que el enfermo no ingirió y precisar la causa, generar indicadores de calidad de los pasos del proceso, confirmar a través de encuesta la satisfacción del usuario, generar informes y proyectos de investigación y culminar con el entrenamiento continuo del personal operante y de nuevos ingresos ${ }^{(25)}$.

La gastronomía clínica es un reto al paradigma de la atención en salud. Termina con el concepto de trabajo individual y gira hacia el trabajo colaborativo, donde todos los miembros son importantes. Incorpora, además nuevos miembros como los gastrónomos y tecnólogos en alimentos con el objetivo de optimizar los 
procesos de preparación de alimentos principalmente en aquellos pacientes que requieren modificaciones dietarias como es el caso del enfermo renal, hepático, con enfermedad celiaca, pacientes con estomas y fístulas gastrointestinales, enfermos con disfagia que reciben papillas o licuados, sin olvidar, al final, el emplatado, o presentación del plato. Por tanto, la prescripción de un plan nutricional es una responsabilidad compartida entre el médico y el nutricionista, el cual deberá ser adaptado por el gastrónomo y cocinero para garantizar una presentación y palatabilidad satisfactoria. De este modo la alimentación de los enfermos deja de ser un acto asistencial para transformarse en una verdadera Terapia Nutricional que supla las necesidades nutricionales individuales.

El término dieta se transforma así en un plan que cubre las necesidades nutricionales, previene la malnutrición por exceso o carencia, detecta interacciones fármaco - nutriente, y evalúa el grado de aceptación del enfermo con la mejor calidad gastronómica, como lo menciona Garriga ${ }^{(25)}$ : "El mejor plato (elaborado de forma impecable en el nivel nutricional y gastronómico) es el que tiene mejor aceptación; es decir, el que se consume por completo". La dieta deja de ser un castigo para convertirse en un proceso terapéutico agradable.

En este cambio de paradigma del servicio de alimentos hay que involucrar a la junta directiva del hospital y sobre todo al área administrativa para darle viabilidad al proyecto ya que puede implicar cambios arquitectóni$\cos$, de equipamiento, capacitación, reingeniería financiera, nuevo personal, etc. Cambiar los paradigmas de las dietas hospitalarias para que ahora estén centradas en el paciente y no en un control administrativo. Es fundamental el conocimiento de la epidemiología del hospital, recursos hospitalarios, organizacionales, gastronómicos y nutricionales, y recursos geográficos y culturales ${ }^{(25)}$ (Tabla 1). El conocimiento de las patologías y la estancia hospitalaria permitirá determinar las características de la dieta basal (cuyo objetivo es mantener un estado nutricional adecuado y prevenir la desnutrición) y la necesidad de dietas especializadas en donde el nutricionista puntualizará los ajustes necesarios.

Existe el menú ya programado que no acepta modificaciones con comida de dos o tres tiempos que con frecuencia se utiliza para hospitales con muchas camas. Resuelve el problema de preparación, pero la calidad del alimento presentado no es del todo satisfactoria, lo que provoca mayor desperdicio ${ }^{(26)}$. Por este motivo se ha propuesto el menú a la carta en donde, previa valoración del nutricionista se le permite al enfermo que escoja algunos de los alimentos lo que ha mejorado la ingesta de alimentos ${ }^{(10,26)}$. Este menú a la carta o más recientemente el sistema de room service con servicio las 24 horas, permite personalizar la dieta y centrarla en el paciente, lo cual es ideal para lograr la satisfacción, aumentar la ingesta y disminuir el desperdicio de alimentos. En este modelo el paciente ordena la comida desde un menú a la carta con alimentos simples y lo recibe en menos de 45 minutos, lográndose que el paciente se empodere de su ingesta de alimentos ${ }^{(26)}$.

\section{Tabla 1. La receta para el mejor plato posible en el hospital}

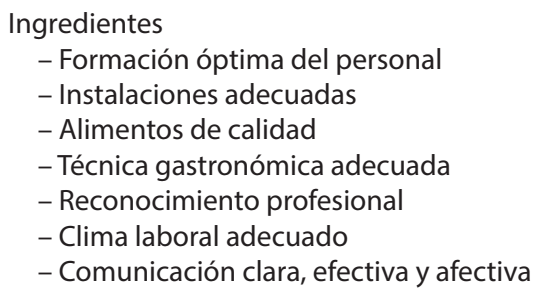

Aptitud y actitud de todo el equipo de salud

Modo de preparación

- Mezclar los siete primeros ingredientes de una manera adecuada, proporcionada y con profesionalidad

- Añadir poco a poco la aptitud de las personas

- Mezclar con sumo cuidado y decorar con una amplia sonrisa

- Al salir, salpicar por encima el cariño de nuestro oficio

Tiempo de cocción

- El tiempo necesario para un correcto acabado del plato

Aparataje

- Las mejores instalaciones posibles

Utensilios

- Nuestras manos, nuestra mente y mucho corazón

Evaluación continua de todo el proceso generando

estándares de calidad

- Registro de actividades

- Generación y evaluación de encuestas de satisfacción

- Evaluación de los desperdicios

- Análisis presupuestal

- Generación de proyectos de investigación.

Tomado y modificado de: Garriga M, Ruiz de las Heras A. Dietética hospitalaria y gastronomía saludable. Nutr Hosp. 2018; 35(No. Extra 4):140-5.

\section{CONCLUSIONES}

La malnutrición hospitalaria sigue siendo un problema con alta prevalencia en los hospitales, principalmente latinoamericanos, favorecida por las condiciones del enfermo, los procedimientos a que es sometido, ayuno deficientemente programado, y por la mala planeación en la preparación y distribución de los alimentos, ignorando 
los hábitos y costumbres del paciente, horarios rígidos e inadecuados, alimentos con temperatura y presentación inadecuadas, que producen resultados conocidos: ingesta deficiente con el consabido deterioro del estado nutricional del enfermo, y gran desperdicio de comida. En resumen, la gastronomía clínica es un cambio del paradigma de los servicios de alimentos en donde, además de una adecuada evaluación médico - nutricional, se optimiza la elaboración de menús, personalizados y acorde con las características del paciente y del hospital, incrementando su consumo, lo que con seguridad propiciará mejoría del estado nutricional de los pacientes y aumentará la satisfacción del servicio de alimentos.

\section{Declaración de conflicto de interés}

No hay conflicto de interés.

\section{Financiamiento}

Ninguno.

\section{Referencias bibliográficas}

1. Allison SP. The management of malnutrition in hospital. Proc Nutr Soc. 1996; 55(3):855 - 62. doi: https://doi. org/10.1079/pns19960084.

2. Avila HD, Bastarrachea MJR, Díaz CA, Flechsig GK, Moedano NG, Norman MO, et al. Atlas cultural de México. Gastronomía. Primera Edición. México: Editorial Planeta; 1988.

3. Real Academia Española. Diccionario de la lengua española [Internet]. 23a edición. 2014. [Fecha de consulta: 30 de mayo de 2020]. Disponible en https://dle.rae.es/

4. Lejavitzer LA. Dieta saludable, alimentos puros y purificación en el mundo grecolatino. Nova Tellus. 2016; 34(1): 109 - 21. doi: http://dx.doi.org/10.19130/iifl.nt.2016.33.2.711.

5. Butterworth CE. The skeleton un the Hospital Closet. Nutr Today. 1974;9:4-8.

6. Swinburne M, Garfield K, Wasserman AR. Reducing Hospital Readmission: Addresing the impact of food security and nutrition. J Law Med Ethics. 2017; 45(suppl 1): 85 -9. doi: https://doi.org/10.1177/1073110517703333.

7. Simzari K, Vahabzadeh D, Nouri S, Khoshbin S, Bektas Y. Food intake, plate waste and its association with malnutrition in hospitalized patients. Nutr. Hosp. 2017; 34(6): 1376 - 81 . doi: https://doi.org/10.20960/nh.1102.

8. Amaral TF, Matos LC, Teixeira MA, Tavares MM, Alvares L, Antunes A. Undernutrition and associated factors among hospitalized patients. Clin Nutr. 2010;29(5):580-5. doi: https:// doi.org/10.1016/j.clnu.2010.02.004.
9. Castillo PJC, Gómez GA, Velasco N, Díaz-Pizarro GJI, Matos A, Mijan de la Torre A. Nutritional Assessment of hospitalized patient in Latin America: association with prognosis variables: The ENHOLA study. Nutr Hospi. 2016; 33(3): 655-62. doi: http://dx.doi.org/10.20960/nh.275.

10. Dupertuis YM, Kossovsky MP, Kyle UG, Raguso CA, Genton L, Pichard C. Food intake in 1707 hospitalised patients: a prospective comprehensive hospital survey. Clin Nutr. 2003; 22(2):115-23. doi: https://doi.org/10.1054/clnu.2002.0623.

11. van Bokhost-de van der Schueren MAE, Roosemalen MM, Weijs JM, Langius JAE. High waste contributes to low food intake in hospitalized patients. Nutr Clin Pract. 2012; 27(2): 274-80. doi: https://doi.org/10.1177/0884533611433602.

12. Valero DA, Caracuel GA. Evaluation of factors affecting plate waste of inpatients in different healthcare settings. Nutr Hosp. 2013; 28(2): 419-27. doi: https://doi.org/10.3305/ nh.2013.28.2.6262.

13. Folgueras TM, Velasco GC, Salcedo CS, Segurola GH, Benítez BN, Ballesteros PMD, et al. Proceso de alimentación hospitalaria. Nutr Hosp. 2019; 36(3):734-42. doi: http://dx.doi. org/10.20960/nh.02543.

14. Alzate A. Comer en el hospital colonial: apuntes sobre la alimentación en tres hospitales neogranadinos a finales del siglo XVIII. Hist Crit. 2012; 46: 18-42.

15. Howard JP. Hospital dietetic and Catering. The science and the art. R Soc Health J. 1979; 99(1): 17-22. doi: https://doi. org/10.1177/146642407909900109.

16. Instituto Tomás Pascual Saenz. Los orígenes de la alimentación hospitalaria en el Siglo XX [Internet]. 15 de mayo de 2012. [Fecha de consulta: 5 de julio de 2020]. Disponible en https://www.institutotomaspascualsanz.com/los-origenesde-la-alimentacion-hospitalaria-en-el-siglo-xx/.

17. Cederholm T, Barazzoni R, Austin P, Ballmer P, Biolo G, Bischoff SC, et al. ESPEN guidelines on definitions and terminology of clinical nutrition. Clin Nutr. 2017; 36: 49-74. doi: https://doi.org/10.1016/j.clnu.2016.09.004.

18. Varela-Moreiras G, Ansón Oliart R, Martínez de Victoria Munoz E. Nutrición, cocina y gastronomía. En: Ángel Gil, editor. Tratado de Nutrición. Tercera edición. Nutrición humana en el estado de salud. Tomo IV: Panamericana; 2017. p. 61931.

19. Achón TM, González GMP, Varela-Moreiras G. Criterios de armonía funcional entre gastronomía y salud: una visión desde la comunidad científica. Nutr Hosp. 2018; 35(No.Extra. 4): 75-84. doi: http://dx.doi.org/10.20960/nh.2131.

20. Duch CG, Duch CC. Gastronomía adaptada al enfermo. Como preparar menús palatables a la vez que nutritivos. Rev Cubana Aliment Nutr. 2017; 27(2): 473-82.

21. Magnoni D. Os segredos do doutor gourmet. Como se alimentar com saúde e muito sabor. Brasil: Matrix editora; 2012.

22. Sígolo RT, Magnoni D, Cukier C, Stikan R. Gastronomía Hospitalar. No conceito do confort food. Brasil: Livraria Balieiro; 2013. 
23. de la Cruz CPJC, Figueredo GR, Dugloszewski C, Ruy DRJAS, Spolidoro NJV, Matos A, et al. Declaración de Cancún: declaración internacional de Cancún sobre el derecho a la nutrición en los hospitales. Nutr Hosp. 2008; 23(5): 413-7.

24. Rodríguez VD. Declaración de Cartagena desde la óptica de la presidencia de la FELANPE. Rev Nutr Clin Metab. 2019; 2(Supl. 1):10-11. doi: https://doi.org/10.35454/rncm. v2supl1.027.
25. Garriga GM, de las Heras A. Dietética hospitalaria y gastronomía saludable. Nutr Hosp. 2018; 35(No. Extra 4): 140-5. doi: http://dx.doi.org/10.20960/nh.2140.

26. McCray S, Maunder K, Crikowa R, MacKenzie-Shalders $\mathrm{K}$. Room Service improves nutritional intake and increases patient satisfaction while decreasing food waste and cost. J Acad Nutr Diet. 2018;118(2):284-93. doi: https://doi. org/10.1016/j.jand.2017.05.014. 